\title{
POSTERIOR AURICULAR NERVE - A NOVEL LANDMARK FOR IDENTIFICATION OF THE FACIAL NERVE IN SUPERFICIAL PAROTIDECTOMY
}

\begin{abstract}
Authors: Arulalan Mathialagan(1), M Ravi Sankar(2)*, Amit Keshri(3), Sabaratnam Mayilvaanan(4)
Authors Affiliations: (1) PDCC Neuro-otology, (2) Associate professor, (3) Additional professor, Neurootology unit, Department of neurosurgery (4) Associate professor, Department of Endocrine surgery, SGPGIMS, Raebareli road, Lucknow, Uttar Pradesh, India
\end{abstract}

\begin{abstract}
Background-Facial nerve identification and preservation is the most critical step in parotid surgery. Though there are described landmarks to locate the facial nerve trunk, they have individual variations. The posterior auricular nerve (PAN) is a branch of the facial nerve and is always present, it can be followed to reach the facial nerve trunk.
\end{abstract}

\section{MATERIALS AND METHODS}

A retrospective cohort study in which analysis of parotidectomy performed from January 2017 to November 2018 at our tertiary referral center was done.

\section{RESULTS}

A total of 23 parotidectomies were performed, of which 18 cases were pleomorphic adenoma. In four cases of pleomorphic adenoma we could clearly identify and preserve the PAN. Using PAN as the landmark the facial nerve trunk was located, all its peripheral branches were dissected and preserved. PAN identification narrows down the target area of dissection to identify the facial nerve trunk.

\section{CONCLUSION}

The posterior auricular branch of the facial nerve can be used as a standard landmark in parotid surgeries, that almost always leads to the facial nerve trunk.

\section{CLINICAL SIGNIFICANCE}

Though identification of PAN may be difficult in all cases, effort must be made to identify it under magnification. If done meticulously PAN can be an ideal landmark to identify facial nerve in parotid surgery.

\section{KEYWORDS}

Parotid surgery, Superficial Parotidectomy, Posterior auricular nerve, Facial nerve.

\section{INTRODUCTION}

Facial nerve identification and preservation is the most critical step in parotid surgery. Though there are described landmarks to locate the facial nerve trunk, they have individual variations. The branch of posterior auricular nerve is a direct branch of the facial nerve is almost always present and leads to the facial nerve trunk, PAN can be identified in parotidectomy dissection and followed to reach it . Material and method

This study was a retrospective cohort study in which analysis of parotidectomies performed from January 2017 to November 2018 at our tertiary referral center was done. We had performed 23 parotidectomy for various pathologies, of which 18 were pleomorphic adenoma.

We have explained a step-by-step parotid dissection for a pleomorphic adenoma involving the tail of the superficial lobe of the right parotid gland, in this dissection we intend to demonstrate the usefulness of the posterior auricular nerve (PAN), a direct branch from the facial nerve trunk, as a definitive landmark in identification of the facial nerve. The nerve directly leads to the facial nerve trunk without any individual anatomical 
variation. All the surgeries were done under surgical loupes with 2.5 magnification for identification of PAN and Facial nerve.For this description of the surgical procedure, we have taken a case of right parotid gland pleomorphic adenoma involving the superficial lobe (Fig 1).

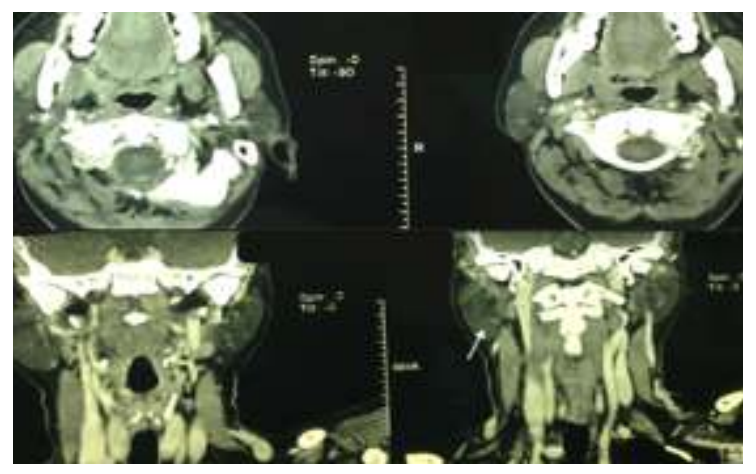

Fig 1 : Pre-operative imaging Contrast enhanced computed tomography showing a nonenhancing mass lesion arising from tail of right parotid gland (white arrow).

\section{Operative Technique}

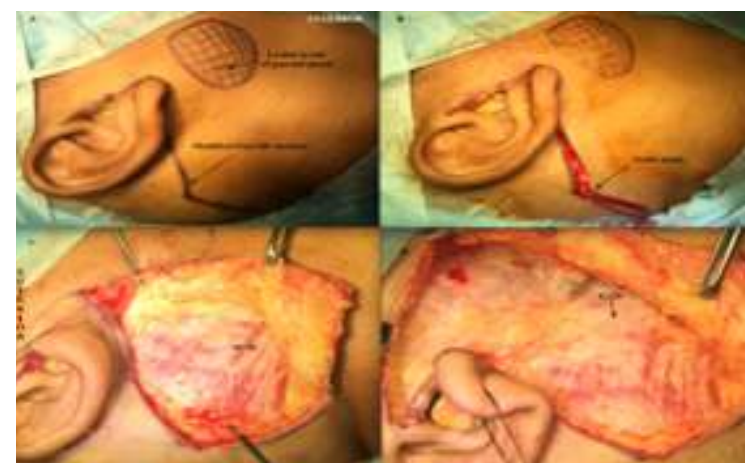

Fig 2 : Incision and skin flap elevation A) Modified face lift incision, B) Skin incision- angled for better approximation, $\mathrm{C}$ ) Neck skin flaps being elevated from deep facial plane of SCM (sternocleidomastoid muscle) lateral to GAN (greater auricular nerve) and D) Skin flap elevation anterior and inferior part along the SMAS (superficial musculo-aponeurotic system) layer and sub-platysmal layer respectively. Fig2Incision and skin flap elevation A) Modified face lift incision, B) Skin incision- angled for better approximation, $\mathrm{C}$ ) Neck skin flaps being elevated from deep facial plane of SCM (sternocleidomastoid muscle) lateral to GAN (greater auricular nerve) and D) Skin flap elevation anterior and inferior part along the SMAS (superficial musculo-aponeurotic system) layer and sub-platysmal layer respectively.

The patient was positioned supine, with extension of neck by placing sandbag in the upper back and the face turned to the left side. A face lift incision- post-auricular incision extending into posterior hairline is made and skin flap is raised in three specific planes- i) supra-perichondrial plane at the tragal cartilage, ii) superficial musculo-aponeurotic plane over the parotid gland and iii) a plane superficial to greater auricular nerve posteriorly and subplatysmal plane anteriorly in the neck (Fig2).

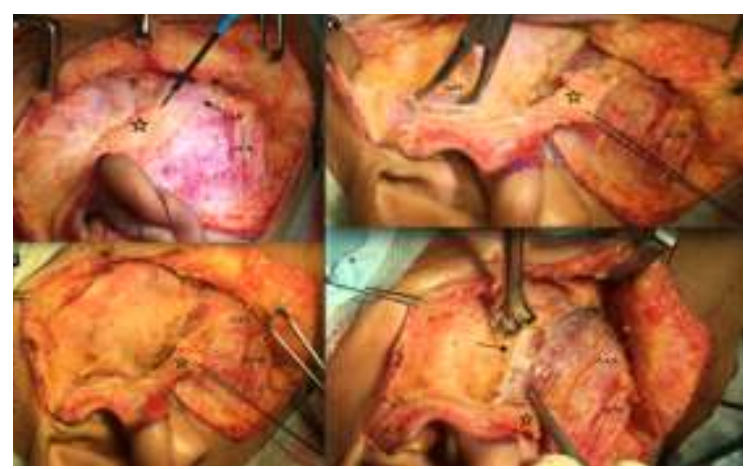

Fig 3 : Dissection of Greater auricular nerve A) Preservation of fibrofatty tissue (star mark) around the greater auricular nerve and its branches with its attachment to the lobule, B) identification of supra-perichondrial plane (SuPP) along the tragal cartilage to locate the tragal pointer, C) the fibrofatty connective tissue around the greater auricular nerve is dissected along with the nerve and retracted laterally in order to preserve the sensory function of the nerve, and D) dissection of the part of superficial lobe (arrow mark) from the sternocleidomastoid muscle.

The fibrofatty tissue over the greater auricular nerve is preserved and dissected off the surface of the sternocleidomastoid muscle and retracted laterally, this step preserves the sensory function of the greater auricular nerve (Fig 3). 


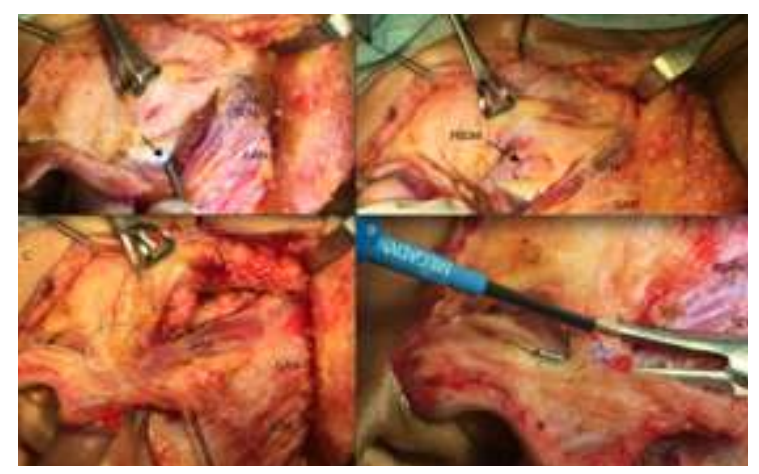

Fig 4 : Delineation of Sternocleidomastoid muscle A) Dissection of the part of superficial lobe from the sternocleidomastoid muscle and retraction of the anterior border of SCM (arrow), B) Identification of the anterior border of upper two-third of sternocleidomastoid muscle and dissected from the parotid tissue and identification of posterior belly of digastric muscle(PBDM), C) Preservation of the connective tissue between the tragal cartilage and anterior insertion of sternocleidomastoid muscle (circle) and D) Meticulous layer-by-layer dissection of the connective tissue between the tragal cartilage and sternocleidomastoid (star mark).

1. Gradually the parotid gland is dissected and retracted anteriorlygaining access medially. The anterior border of sternocleidomastoid muscle is delineated and is retracted, followed by identification of the posterior belly of digastric muscle and its superior border. The tragal pointer deeper in the supra-perichondrial plane is identified.The connective tissue between the tragal pointer and the SCM is preserved and this is the site were the posterior auricular nerve (PAN) will be present (Fig 4).

2. PAN is identified running in the tympanomastoid suture area, deeper in the connective tissue. The PAN predicts the possible site of facial nerve trunk. The PAN directly leads to the facial nerve trunk as it is one of branches from the facial nerve near the stylomastoid foramen. Dissection is started from the superior border of posterior belly of digastric towards the predicted location of the facial nerve trunk (Fig 5).

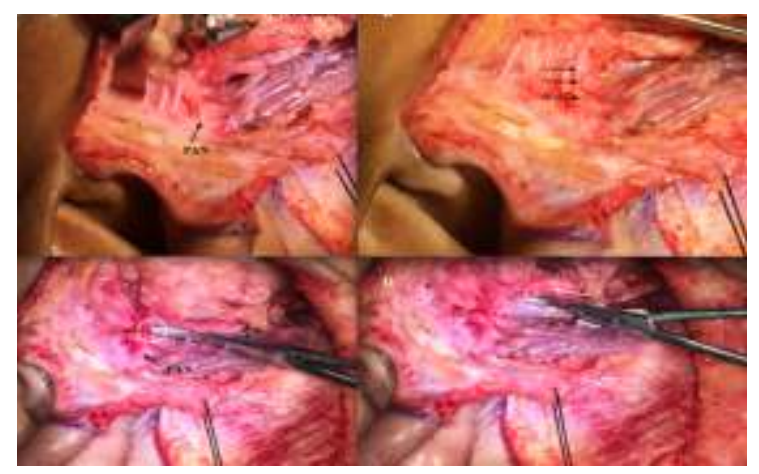

Fig 5 : Identification of the PAN A) Identification of the Posterior auricular nerve (PAN) in the tympano-mastoid suture region, B) Meticulous dissection and further delineation of the PAN (arrows), C) locating the possible site of facial nerve trunk based on the direction of the PAN(circle), and D) Dissection started from the superior border of posterior belly of digastric towards the predicted location of the facial nerve trunk.

The PAN pin-points the location of the facial nerve trunk at the area medial to the mastoid process between the tragal pointer and the superior border of digastric (Fig 6).The major divisions of the facial nerve are dissected from the superficial lobe of the parotid gland and the terminal branches are separated from the superficial lobe by sharp dissection.Superficial lobe is resected, and all the branches are clearly identified (Fig 7). Hemostasis is secured using bipolar electrocautery. Incision is closed in two layers,a subcutaneous layer, and a cutaneous layer by intermittent sutures afterplacing a suction drain.

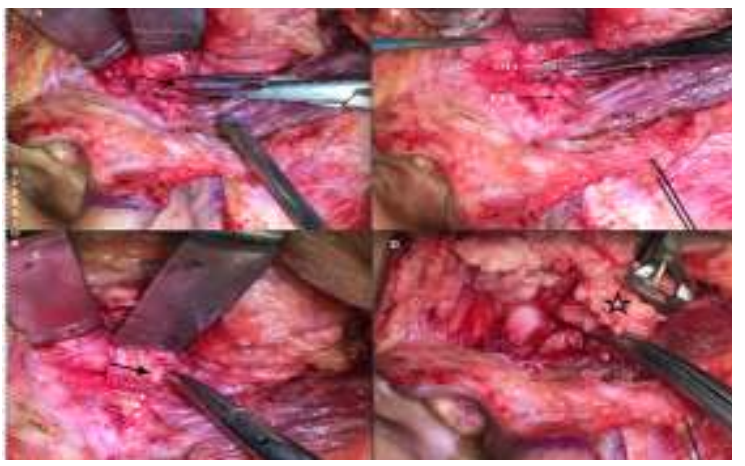

Fig 7 : Identification of the terminal branches of Facial nerve A) Identification of the Buccal branch 
of the facial nerve, B) Identification of the Marginal mandibular branch of the Facial nerve, C) After removal of the tumor, the facial nerve trunk, its upper and lower divisions and all the branches of the lower division are visualized and D) Wound closure in two layers with a suction drain (white arrow).

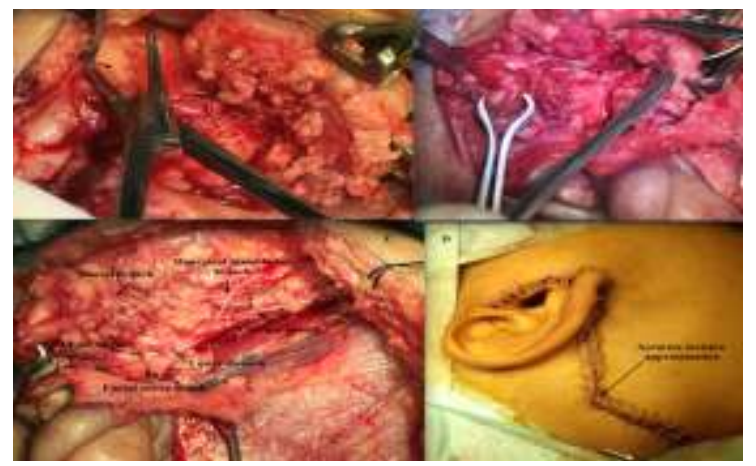

\section{RESULTS}

A total of 23 parotidectomies were performed, of which 18 cases were pleomorphic adenoma. In four cases of pleomorphic adenoma, we could not identify and preserve the PAN. Using PAN as the landmark, the facial nerve trunk was located in the rest of the 14 cases; all its required peripheral branches were dissected and preserved.

\section{DISCUSSION}

Identification and preservation of the facial nerve is the most vital step in parotid surgery. There are surgical landmarks to be identified during dissection of the parotid gland, which aids in locating the nerve trunk as it exits the stylomastoid foramen. The tragal pointer, the most prominent part of the cartilaginous external auditory canal, the superior border of the posterior belly of digastric muscle are the most often used surgical landmarks. Other described landmarks are mastoid tip, stylomastoid artery, styloid process and tympano-mastoid suture line. These landmarks are constantly present in all the patients, but their relation with the facial nerve trunk is variable. Liu et al. (4) has described the usefulness of the Posterior auricular artery and nerve in a cadaveric study. Meticulous dissection and identification of the PAN under surgical loupes aids in precisely identifying the facial nerve trunk without any difficulty. There is no individual variation in the relation of the PAN and facial nerve trunk. Figure 8 depicts the relation of the PAN with the main trunk of the Facial nerve in three different cases. Identifying the PAN may be challenging due to its slender calibre; with stepwise dissection, its identification can be made in all cases. Use of surgical loupe or microscope is mandatory as the PAN is very slender, and often in the surgical field, it is difficult to differentiate it from the fibrous strands by the naked eye. Figure 8 shows the relation of PAN and Facial nerve trunk

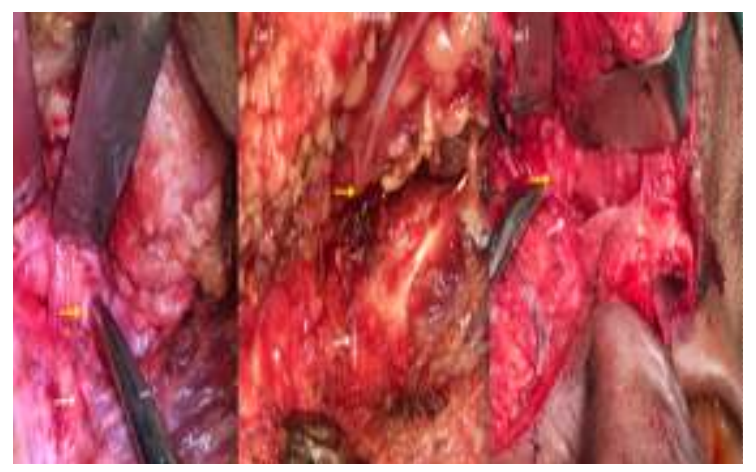

Fig 8 : Relation of PAN (white arrow) and facial nerve trunk (yellow arrow) in three different cases.

For a superficial parotidectomy, modified Blair's incision is usually used, but a modified facelift incision can also be used in tumours involving only the tail of the gland. The facelift incision is cosmetically more acceptable, as the surgical scar is behind the pinna and along the hairline, thus almost hidden.

\section{CONCLUSION}

The typical landmarks described in the literature have individual variation concerning the distance to the facial nerve trunk, while the posterior auricular nerve, a branch of the facial nerve itself, invariably leads to the nerve trunk. In some cases, it may be very thin and thus may be difficult to identify and delineate. An attempt to identify PAN should be made, and a stepwise meticulous dissection and identification of all the landmarks along with PAN will almost always 
help identify facial nerve trunk, and it is narrow down the search of facial nerve trunk more preciously.

\section{REFERENCES}

1. N. Pather, M Osman (2006) Landmarks of the facial nerve : implications for parotidectomy. Surg Radiol Anat 28:170-5.

2. Blau I, Vaisbuch Y, Marom A (2019) A New Method for Tracing the Facial Nerve Trunk Using the Posterior Auricular Nerve. Clin Anat 32(3):453-7.

3. Righini C (2012) Facial nerve identification during parotidectomy. Eur Ann Otorhinolaryngol Head Neck Dis 129(4):214-9.

4. M Liu, S Wang, A Benet et al(2018) Posterior auricular artery as a novel anatomiclandmark for identification of the facial nerve : A cadaveric study. Head and Neck 40(7):1461-1465.

\section{${ }^{*}$ Corresponding author:}

M. Ravi Sankar

MSDNB

Associate professor, Neuro-otology unit, Department of neurosurgery,

C-Block, SGPGIMS,

Raebareli Road, Lucknow, Uttar Pradesh, India.226014.

M: 8004221416, 7010599665

drravisankarpgi@gmail.com 